Hans-Otto Rosenbohm

\title{
Äquivalentlose Lemmata und kulturgebundene Wörter in zweisprachigen Wörterbüchern
}

(Der nachstehende Aufsatz ist das leicht überarbeitete Manuskript eines Vortrages auf der ADLA-Jahrestagung 1990 in Kopenhagen)

"Amtsborgmesteren spiser frokost på døgneren" - mit Hilfe dieses aus dänisch-landeskundlicher Sicht zwar möglichen, wenn auch unwahrscheinlichen Tatbestandes soll versucht werden, einige Probleme der Präsentation solcher Wörterbucheinträge $\mathrm{zu}$ beleuchten, die sich der 1:1-Gleichung "Lemma A(usgangssprache) = Lemma Z(ielsprache)" zur Verzweiflung der Lexikographen und zum Schaden der Wörterbuchbenutzer hartnäckig entziehen.

\section{Definitionen ...}

\section{a. Wörterbücher}

Unter "zweisprachigen Wörterbüchern" werden hier (zumindest primär) Hin-Übersetzungswörterbücher oder aktive Wörterbücher verstanden, d.h. Wörterbücher, die darauf abzielen, es einem kompetenten Sprecher der Ausgangssprache zu ermöglichen, das Lemma möglichst ohne Informationsverlust in die Zielsprache zu übersetzen. Anders gesagt: Es geht darum, einen ausgangssprachlich kodierten Text, die Information L1, in einen zielsprachlichen Text, die Information L2, umzusetzen, wobei diese Information invariant zu halten ist. Nun weiß jeder Übersetzer, d.h. im Grunde jeder Wörterbuchbenutzer, daß dies in der Praxis erhebliche Probleme aufwirft, da Translation im Gegensatz zur Mathematik nur begrenzt an die Gültigkeit einfacher Gleichungen glauben darf. Erinnert sei an die goldene Regel des Lateinlehrers: "So wörtlich wie möglich, so frei wie nötig" — wobei natürlich "wörtlich" und "frei" niemals definiert wurden.

\section{b. Äquivalentlose Lemmata}

Es mag semantische Felder geben, in denen volle Äquivalenz vorhanden ist. Zu denken wäre z.B. an weite Bereiche der Sprache der Technik, da man davon ausgehen kann, daß Bezeichnungen für genau definierte 
Konkreta (d.h. technische Komponenten) und Funktionsweisen (in Form von Funktionsverben) erstens in beiden Sprachen existieren und zweitens inhaltlich invariant sein müssen, da der technische Gegenstand bzw. Vorgang außersprachliche Identität aufweist.

(Beispiel:
DK: rundballepakker
D: Rundballenwickelmaschine;
DK: vendeplov
D: Volldrehpflug usw.)

Dieses Prinzip wird nicht dadurch erschüttert, daß in manchen Bereichen zwischen bestimmten Sprachen kein eigensprachliches Äquivalent vorhanden ist, sondern das fremdsprachliche Lemma (manchmal versehen mit einheimischer Grammatik) übernommen wird

(Beispiel:
E: harddisk
DK: harddisk(en)
D: Festplatte;
E: interface
DK: interface $(\mathrm{t})$
D: Schnittstelle).

In anderen semantischen Bereichen besteht eine solche Übereinstimmung nicht. Die Rechts- und Verwaltungssprache z.B. spiegelt die jeweiligen Systeme wider, die eben nicht identisch sind. So operiert beispielsweise das dänische Recht nur mit zwei Formen des ehelichen Güterstandes, nämlich
DK: fælleseje
DK: særeje
D: Gütergemeinschaft und
D: Gütertrennung,

während der gesetzliche Güterstand des deutschen Rechts, die

\section{D: Zugewinngemeinschaft $\quad$ DK: -}

im dänischen Recht unbekannt ist und daher natürlich im Dänischen kein Äquivalent vorliegt.

D: Zugewinngemeinschaft ist somit ein typisches Beispiel für ein “äquivalentloses Lemma”.

Noch problematischer sind Begriffe, bei denen auf den ersten Blick eine Oberflächen-Identität vorzuliegen scheint:

DK: landsret

D: Landgericht.

In Wirklichkeit gibt es juristische Unterschiede bei Instanzenzug und Zuständigkeit, die — zumindest in bestimmten Fällen — eine Gleichsetzung nicht zulassen.

Im gesamten Bereich der Verwaltung gibt es zahllose Bezeichnungen, die einen ganz bestimmten Sachverhalt ausdrücken, der oft in etwa auch im anderen Sprachbereich existiert: 
DK: amtskommune

DK: amtsborgmester

DK: energiattest untere dänische Verwaltungseinheit;

etwa: Landkreis

politischer und administrativer Leiter

einer "amtskommune";

etwa: Kreispräsident bzw. Landrat.

Bescheinigung über durchgeführte

Wärmedämmung in Wohnhäusern

usw. usw.

\section{c. "Kulturgebundene Wörter"}

Auch außerhalb mehr oder weniger klar abgegrenzter semantischer Bereiche wie Jura und Technik gibt es Begriffe, die in ihrer jeweiligen Kultur gebunden sind ("kulturgebundene Wörter"). Darunter sind Wortbildungen zu verstehen, die einen Sachverhalt "anders" ausdrücken als in anderen Sprachen bzw. Gegebenheiten beschreiben, die es in anderen Kulturen "so" nicht gibt. Als Beispiel aus dem Dänischen seien genannt:

DK: døgn (Zeitraum von 24 Stunden);

DK: døgner (sl. rund um die Uhr geöffneter Kiosk)

DK: frokost (meist kalte Mahlzeit um die Mittagszeit)

DK: (vb.) at jule (weihnachtliche Aktivitäten betreiben),

DK: gruk (Kunstwort für eine besondere Gedichtform des Dichters und Philosophen Piet Hein);

DK: grønsmutte (10-12jährige Pfadfinderin (assoziativ mit Vogelnamen auf ...smutte verbunden).

Eine Sondergruppe sind Wörter, zu deren einzelnen Elementen jeweils Äquivalente vorliegen, die jedoch als Gesamtheit in ganz bestimmter Weise angewandt werden:

DK: whiskybælte

DK: giftskatter

DK: efterskole
D: * Whiskygürtel (wohlhabende nördliche Vororte von Kopenhagen);

D * Giftsteuern (Steuern auf Genußmittel wie Alkohol und Tabak);

D: * Nachschule (Internatschule, in der die Klassen 8. - 10. der dänischen "Volksschule" absolviert werden können).

\section{... und die Folgen für das Wörterbuch}

\section{a. Artikelstruktur}

Die Forderung nach invarianter Informationsübertragung beim Übersetzen hat erhebliche Konsequenzen für den Wörterbucheintrag, bzw. den Artikelaufbau in aktiven Wörterbüchern. So kann man immer dann 
auf analytische, synonymische usw. Bedeutungsdefinitionen verzichten, mit denen z.B einsprachige Wörterbücher arbeiten, wenn ein Äquivalent vorhanden ist und die Bedeutungsinhalte, einschließlich eventueller Polysemien, deckungsgleich sind. Andererseits sind Bedeutungstrennungen unerläßlich, sobald das semantische Feld L1 nicht dem semantischen Feld L2 entspricht — was leider eher die Regel als die Ausnahme ist.

Beispiel:

$\mathrm{DK} \rightarrow \mathrm{D}:$ and $\rightarrow$ Ente

nämlich in beiden Sprachen 1. Vogel und 2. Falschmeldung; dagegen umgekehrt

$\mathrm{D} \rightarrow \mathrm{DK}$ : Ente 1. and (auch Zeitungs-) 2. (Uringefäß für bettlägerige Männer) urinflaske; 3. kalte $\sim$ punch; 4. (Autotyp Citroën $2 \mathrm{CV}$ ) $2 \mathrm{CV}$.

Auf die Erörterung weiterer Implikationen der Sonderung zwischen aktiven und passiven Wörterbüchern, so z.B. notwendige oder wünschenswerte bzw. überflüssige grammatische Erläuterungen, Aussprache, Etymologie usw. sei hier verzichtet. (1)

\section{b. "Wort" und Lemma im Wörterbuch}

Äquivalentlose Lemmata und kulturgebundene Wörter haben gemeinsam, daß sie einem und nur einem Sprachsystem angehören. Daher wird im folgenden auf eine weitere Sonderung verzichtet.

Ohne die benutzten Ausdrücke "Wörter" und "Lemmata" weiter zu diskutieren, sei soviel gesagt: Unter "Wörtern" sind die konkret in einem beliebigen Text auftretenden Größen zu verstehen, d.h. Wortformen, Ableitungen und Zusammensetzungen, die im Wörterbuch bestenfalls zum Teil aufgenommen sind. (DK: “ænder", also der pl. von DK: and — D: Ente ist mir in keinem Wörterbuch begegnet). "Lemma" dagegen ist das Stichwort, das im Wörterbuch (das also eigentlich "Lemmabuch" heißen müßte) abstrahiert und halbfett in einer Grundform auftaucht.

Dies ist die prinzipielle Crux des Lexikographen: das Wörterbuch zerlegt Texte in Wörter — oder schlimmer: Lemmata - und nimmt dabei den Wörtern ihre Textualität, durchaus im Widerspruch zu allgemein akzeptierten Prinzipien der modernen Linguistik und Übersetzungslehre.

Lemmatisierung für die Zwecke des Wörterbuches bedeutet also immer die Herauslösung eines Wortes aus seinen (text)grammatischen und (text)semantischen Bindungen. 
Ein interessanter Gedanke in diesem Zusammenhang wäre die Arbeit mit Konkordanzen, die, soweit ich sehe, Wörterbücher ergänzen könnten, jedoch für die mir bekannten Sprachen nur bruchstückhaft vorliegen.

\section{c. Sem und Semem}

Ein Blick auf "Sem" und "Semem" ist hilfreich in unserem Zusammenhang: Mit Neubert (2) sei unter "Sem" ein "Bedeutungsatom" verstanden, also z.B. "rot" usw. Ein "Semem" wäre danach ein "Bedeutungsmolekül", d.h. die charakteristische Kombination von Semen, die einer bestimmten Sprache eigentümlich ist.

Das Charakteristische betrifft zwei Bereiche: zum einen mehrfache semantische Füllung eines Lexems: "rote Fahne", "rote Brigaden", "Rotfront" ist etwas anderes als "rotes Blut" oder "rote Jacke" — ganz zu schweigen von den konnotativen Implikationen in verschiedenen Gesellschaftsformen oder zu verschiedenen Zeiten. "Rot" war — zumindest in nicht allzu ferner Vergangenheit — im offiziellen DDR-Sprachgebrauch durchaus positiv besetzt. Das Gegenteil war (und ist) der Fall in der Bundesrepublik.

Das zweite Charakteristikum - und hier wird es für den Lexikographen erst richtig interessant - ist die Koppelung mit anderen Semen, die ein Semem ergibt, das nur in einem bestimmten Kulturkreis verständlich oder logisch ist. Die natürliche Sprache ist nun einmal ein historisch und sozial determiniertes Phänomen. Um im Farbbereich zu bleiben: In einem dänisch-deutschen Wörterbuch ist es natürlich unproblematisch "rød" mit "rot" wiederzugeben, weniger klar wäre die Sachlage in einem deutsch-apachischen Wörterbuch, da bestimmte Indianerstämme in der Wüste von Arizona das Farbspektrum anders einteilen als Mitteleuropäer. Und wie ist es mit dem dänischen Ausdruck "postkasserød", der sich auf die hübsche Farbe dänischer Briefkästen bezieht — für einen Deutschen jedoch, der gelbe Briefkästen kennt, wäre die Bezeichnung "briefkastenrot" zumindest überraschend. Die funktional äquivalente Wiedergabe im Deutschen wäre ohne Zweifel "feuerwehrrot" — aber welcher Lexikograph wagt es, über den Schatten seiner Zweisprachigkeit zu springen: Es könnte vielleicht jemand auf den Gedanken kommen, daß DK: postkasse im Deutschen D: "Feuerwehr" heißt ....

Es ließen sich weitere Beispiele anführen: "hautfarben" ist sicher problematisch in den Wörterbüchern Deutsch-Suahili oder Deutsch-Chinesisch, "auberginefarben" setzt voraus, daß man Auberginen kennt usw. usw. 


\section{Die Intuition des Lexikographen — oder ...?}

Aus dem Gesagten ergibt sich: Bei der Gegenüberstellung zweier einzelsprachlicher Systeme ist idealiter ein gemeinsamer Bezugspunkt nötig, ein Tertium comparationis. Der Lexikograph geht folgerichtig dennoch wohl meist unbewußt - von der "Außereinzelsprachlichkeit von Begriffen" aus, von bestimmten "Archisemem", die die Einzelsprachen übergreifen. Das ist sicher in vielen Fällen möglich und richtig (rot als Farbe des Blutes oder des Feuers - wie man in zahlreichen einsprachigen Bedeutungswörterbüchern nachlesen kann), gilt aber, wie sich aus den wenigen Beispielen ergab, keineswegs generell.

Ein weiteres Problem, das meist intuitiv gelöst wird, ist die fehlende oder unvollständige Vergleichbarkeit der syntagmatischen Ebene. Wenn man akzeptiert, daß sich prinzipiell alles in allen Sprachen ausdrücken läßt, muß man sich klarmachen, daß nicht alle Sprachen auf dieselbe Weise ökonomisieren. In einigen wird ein komplexer Zusammenhang, z.B. eine Bewegung, in einem einzigen Verb ausgedrückt, während andere Sprachen syntagmatisch vorgehen: (Beispiel: D: tätscheln — NL: strelende klopjes geven). Meines Wissens gibt es für keine Sprache semasiologische Analysen in ausreichendem Umfang, die es dem zweisprachigen Wörterbuchmacher erlauben würden, durch ein umgekehrtes onomasiologisches Verfahren das auch theoretisch korrekte Äquivalent zu extrahieren.

Als dritter Punkt, der sich unmittelbar aus dem zweiten ergibt, sei die semantische Feldanalyse angesprochen. Wer Fremdsprachen unterrichtet, kennt zahllose Beispiele, daß insbesondere Lerner ("Learner") oft das Wörterbuch benutzen wie der Teufel die Bibel: Hans karakterer er slette — seine Charaktere sind ebene; Det er galt — das ist verschnittener Eber — Beispiele, die dänischen Deutschlehrern bekannt sind. In vielen Fällen ist der Schüler jedoch exkulpiert, da ihn das Wörterbuch im Stich läßt und einfach drei, vier oder noch mehr Äquivalente nebeneinander angibt; (Bork: DK adskillige - D mehrere, etliche, manche; DK adskillelse D Trennung, Sonderung, Teilung, Scheidung) - so tuend, als handele es sich hier um Synonyme, die unterschiedslos verwendbar wären. Erfahrungsgemäß wählt der Lerner in $90 \%$ der Fälle das erstgenannte (was in Klammern bemerkt natürlich beim Artikelaufbau eines Wörterbuches berücksichtigt werden könnte (3)). Hier würde eine Darstellung der Distribution dabei helfen können, die bisher oft intuitive Identifikation bei der Transposition mit ihrer recht bescheidenen Trefferquote auszuschalten. 


\section{"Die Wahrheit ist konkret ..."}

Im folgenden sollen nun die aufgezeigten Probleme und Prinzipien an einem Beispiel aus den in Dänemark geläufigen Wörterbüchern illustriert werden. Wir wählen das Lemma DK amt mit Komposita in 5 dänischfremdsprachigen Wörterbüchern (für das Englische und Französische gibt es jeweils ein großes Wörterbuch und ein bescheideneres einbändiges Wörterbuch, die nicht (zumindest nicht direkt) voneinander abgeleitet sind. Für das Deutsche dagegen gibt es bislang nur ein Wörterbuch, das vom Umfang her zwischen den beiden Wörterbüchern der anderen Sprachen liegt).

\section{Dänisch - Deutsch}

Dansk-tysk Ordbog. Af Egon Bork. 9. udgave. København (Gyldendal) 1987.

\section{Dänisch - Englisch}

2.1. Dansk-engelsk Ordbog. Af Jens Axelsen. Niende udgave, 3. oplag. København (Gyldendal). 
118

2.2. Hermann Vinterberg og C.A. Bodelsen Dansk-engelsk Ordbog. Anden reviderede og udvidede udgave. (I: A-Måttevarer). København (Gyldendal).

\section{Dänisch - Französisch}

3.1. Dansk-fransk Ordbog. Af N. Chr. Sørensen. 5. udgave. København (Gyldendal) 1983.

3.2. Andreas Blinkenberg og Poul Høybye: Dansk-fransk Ordbog. Tredje reviderede og forøgede udgave. Bd. 1: A-L. København (Nyt Nordisk) 1975. 


\section{"Amt" und "amtskommune"}

Seit 1970 ist Dänemark in 14 "Amtskommunen" eingeteilt, die zwischen 11 und 32 "Primärkommunen" umfassen. (Kopenhagen und Frederiksberg haben einen Sonderstatus). Die Einwohnerzahl schwankt zwischen 47.000 und 630.000, die Fläche zwischen 421 und 6.171 qkm. Die "Amtskommunen" haben vom Volk gewählte Vertretungen mit dem "Amtsbürgermeister" an der Spitze. Ihre Hauptaufgaben sind der öffentliche Service gegenüber dem einzelnen Bürger, die Trägerschaft für Schulen (insbesondere Gymnasien), Krankenhäuser und andere Institutionen sowie die technische Infrastruktur, darunter der öffentliche Verkehr. (4)

Wie gehen nun die Wörterbücher diesen recht komplexen Zusammenhang an?

Es würde zu weit führen, alle 5 Wörterbuchartikel im einzelnen zu diskutieren, daher nur einige prinzipielle Bemerkungen und eine etwas eingehendere Behandlung des Artikels im dänisch-deutschen Wörterbuch, insbesondere zum DK amt, DK amtskommune und DK amtsborgmester.

Alle Wörterbuchautoren sind sich der Tatsache bewußt, daß es sich hier um äquivalentlose Lemmata handelt, die in keiner der Zielsprachen eine hundertprozentige Entsprechung haben. Daher sind in allen Fällen Hinweise nötig. Bork verweist unter amt auf amts-kommune oder statsamt - was sicher nicht bedeuten soll, daß es gleichgültig ist, wo man nachschlägt, da die beiden dänischen Institutionen durchaus unterschiedlich sind. Der Eintrag unter statsamt allerdings legt genau dies nahe: "omtr (= etwa) Regierungsbezirk, Kreis" — ohne weitere Erläuterung. Beide Äquivalente kommen auch unter amtskommune vor.

Die beiden englischen und die beiden französischen Wörterbücher geben Geschlecht und Pluralis des dänischen Lemmas an, was darauf schließen läßt, daß sie auch Ambitionen als passive Wörterbücher haben. Beide englische fügen hinzu: "kan gengives" (= läßt sich wiedergeben) - eine unglückliche Formulierung, die wohl kaum als Trost gemeint sein dürfte, sondern wohl bedeutet "Läßt sich eigentlich nicht wiedergeben, aber wenn es denn sein muß ...”. Vinterberg-Bodelsen haben außerdem eine (unzureichende) Bedeutungsdefinition in der Zielsprache und einen Realkommentar, der besagt, daß wegen der Unterschiede der englischen (wie steht es mit der amerikanischen und der anderer englischsprachiger Länder?) und der dänischen "Lokalverwaltung" die dänischen Komposita mit "amt" nur annäherungsweise wiedergegeben werden können. Nimmt man "amtskommune", die die offizielle Bezeichnung für das 
umgangssprachliche "amt" ist, hinzu, geben die englischen Wörterbücher insgesamt fünf verschiedene "Äquivalente": county, county council district, shire, county (borough).

Sørensen benutzt unter amt den gleichen unglücklichen Hinweis "kan gengives:" wie die englischen, unter amtsborgermester inkonsequent "(omtr.)" (= etwa). amtskommune fehlt — Lemmalücke.

Blinkenberg-Høybye machen als einziges Wörterbuch auf die Möglichkeit aufmerksam, den dänischen Begriff in der Zielsprache als "amt" zu übernehmen. Hinzu kommt ein Realkommentar unter amt und unter amtskommune, der auf engem Raum erstaunlich viele Fakten aufzählt. Diese Definition wird dann unter amtsborgmester wieder aufgegriffen, der als "maire d'une "amtskommune"" definiert wird. Schließlich erhält man den Annäherungswert "département [danois]". Insgesamt sei schon hier gesagt, daß diese Art der Behandlung eindeutig den höchsten (und korrektesten) Informationswert aller untersuchten Wörterbücher hat.

In dieselbe Richtung zielt Bork, allerdings mit weniger Erfolg. Unter amtskommune wird angegeben: "(stor (= großer) Regierungsbezirk" mit dem zusätzlichen, für die Übersetzung irrelevanten Hinweis, daß 6 Bundesländer in 2-6 aufgeteilt sind — "Landkreis .., (mindre (= kleiner)) Gemeindeverband, in Rheinland-Pfalz jedoch Verbandgemeinde; (in der DDR) Bezirk. Mit diesen Informationen läßt sich nichts anfangen: Soll man die "Amtskommunen" Roskilde, Bornholm und Kopenhagen (unter $1.000 \mathrm{qkm}$ ) mit "Gemeindeverband" und die anderen nach Gusto mit Regierungsbezirk oder Landkreis übersetzen? Oder soll man für rheinland-pfälzische Leser "Verbandgemeinde" und für ein DDR-Publikum "Bezirk" benutzen? Dasselbe wiederholt sich bei amtsborgmester. In diesem Licht macht es weniger, daß mehrere der Eintragungen eindeutig falsch sind, nämlich Regierungsbezirk (staatliche Aufsichtsbehörde ohne Volksvertretung) bzw. Regierungspräsident (ein Beamter, kein Volksvertreter) und Oberkreisdirektor (ebenfalls ein Beamter, nämlich der Verwaltungschef einer “Amtskommune"). Letzterer, der auf Dänisch amts(kommunal)direktør heißt, wird von Bork ebenfalls fehlerhaft als "omtr. Verbandsbürgermeister" wiedergegeben, obwohl eine zureichende Äquivalenz zu D: Oberkreisdirektor vorliegt.

Bei Bork zeigt sich sehr deutlich ein prinzipielles Problem vieler Wörterbücher: die fehlende Sonderung zwischen aktivem und passivem Wörterbuch. Bork, der seine Artikel im Grunde aktiv anlegt (z.B. sichtbar am Verzicht auf jede Form von ausgangssprachlicher Grammatik), gibt nicht Hin-Übersetzunghilfen, sondern einen Strauß mehr oder weniger 
falscher Her-Übersetzungsvorschläge, unter denen sich ein deutscher Muttersprachler (wenn man von Schweizern und Österreichern absieht), das ihm geläufigste heraussuchen kann.

Übrigens haben auch Vinterberg-Bodelsen ein interessantes Beispiel eines Typs, der in vielen Wörterbüchern zu finden ist: In Dänemark und damit auch in dänischen Texten gibt es kein amtsfængsel ("Amtsgefängnis"), wohl aber kommt in jedem Western das "county jail" vor — also eine typische Rückübersetzung, die in einem aktiven Wörterbuch nichts zu suchen hat.

\section{Schlußfolgerungen}

Genug der Kommentare. Welche Forderungen ergeben sich nun daraus an ein aktives Wörterbuch in bezug auf äquivalentlose Lemmata und kulturgebundene Wörter?

Einleitend wurde gesagt, daß Wörterbücher kaum umhin können, die Wörter durch die Abstraktion auf Lemmata ihrer Textualität zu berauben. Die Forderung muß also ganz einfach sein, daß ein Wörterbuchartikel dem Lemma soviel Text wie möglich beigibt, sei es durch Realkommentare oder durch Beispiele.

Hinzuzufügen wäre, daß auch der Kontext bzw. der Zweck der Übersetzung die Wahl des Äquivalents beeinflussen kann (und sollte). An einem einfachen Beispiel verdeutlicht (nach Koller (5)):

D: Bundesgerichtshof kann je nach Übersetzungtyp auf verschiedene Weisen z.B. im Dänischen wiedergegeben werden:

1. Beibehaltung des ausgangssprachlichen Lemmas: DK: "Bundesgerichtshof"

2. Lehnübersetzung: DK: forbundsdomstol

3. beschreibende Übersetzung: DK: Vesttysklands øverste domstol

4. adaptierende Übersetzung: DK: den vesttyske højesteret.

Typ 1 käme in Frage für Juristen, denen der Begriff geläufig ist, Typ 3 oder 4 z.B. für einen Zeitungstext, der auf juristische Genauigkeit keinen Wert zu legen braucht, usw.

Der Artikel Bundesgerichtshof im deutsch-dänischen aktiven Wörterbuch müßte also ausreichende Informationen zur Verfügung stellen, um diese Wahl zu ermöglichen.

Blinkenberg-Høybye kommen der Sache recht nahe, verzichten allerdings auf übersetzte Beispiele. 
Aus dem oben Ausgeführten ergeben sich eine Reihe von Forderungen an aktive zweisprachige Wörterbücher. Kurz nach dem Erscheinen dieses Artikels wird in Dänemark ein neues dänisch-deutsches aktives Wörterbuch erscheinen (Munksgaards Dansk Tysk Ordbog), in dem zumindest versucht wird, einige der angeschnitteten Probleme ein wenig besser zu lösen.

Um den geneigten Leser, der bisher geduldig auf einen Übersetzungsvorschlag für den einleitenden Satz geharrt hat, nicht zu enttäuschen, seien hier - keineswegs erschöpfend gemeinte - Vorschläge für die entsprechenden Artikel wiedergegeben. Einige Erläuterungen vorweg: (DK) signalisiert "äquivalentloses Lemma” bzw. "kulturgebundenes Wort"; die Doppeltilde drückt aus, daß es sich bei den angegebenen Äquivalenten um Näherungswerte handelt; in eckigen Klammern stehen Realkommentare in der Zielsprache: (+ gr) gibt hier nur an, daß an dieser Stelle die grammatischen Angaben stehen, auf die die Verfasser sich verständigt haben. Im "Schwanz" des Artikels (hinter*) kommen dann Beispiele, die erstens dem Lemma möglichst viel Textualität beigeben sollen und zweitens möglichst authentisch sind.

Man versuche sich selbst an der Übersetzung — die Probe aufs Exempel, ob ein aktives Wörterbuch seinen Zweck erfüllt! 


\section{Literatur}

(1) siehe dazu: Kromann/Riiber/Rosbach: Betydningsbeskrivelse og ordbogstyper inden for tosprogs-leksikografien med særligt henblik på en dansk-tysk ordbog. ARK 1, 1979 (København).

(2) Albrecht Neubert: Zu einigen aktuellen Problemen der lexikalischen Semantik. Berlin 1981.

(3) siehe dazu: Anette Grindsted: Principper for præsentation af ækvivalenter i oversættelsesordbøger. Odense 1988.

(4) Amtskommunen. Udgivet af Amtsrådsforeningen. 1978.

(5) Koller: Einführung in die Übersetzungswissenschaft. Heidelberg 2.1983. 
124 